\title{
Properties of Hydrogenated Nanoporous SiC: An Ab Initio Study
}

\author{
Blair R. Tuttle, ${ }^{1,2}$ Nathan J. Held, ${ }^{1}$ Lai Hin Lam, ${ }^{1}$ \\ Yu-Yang Zhang, ${ }^{2}$ and Sokrates T. Pantelides ${ }^{2,3}$ \\ ${ }^{1}$ Department of Physics, Penn State Behrend, Erie, PA 16563, USA \\ ${ }^{2}$ Department of Physics and Astronomy, Vanderbilt University, Nashville, TN 37235, USA \\ ${ }^{3}$ Oak Ridge National Laboratory, Oak Ridge, TN 37831, USA
}

Correspondence should be addressed to Blair R. Tuttle; brt10@psu.edu

Received 4 November 2016; Accepted 20 December 2016; Published 10 January 2017

Academic Editor: Victor M. Castaño

Copyright (C) 2017 Blair R. Tuttle et al. This is an open access article distributed under the Creative Commons Attribution License, which permits unrestricted use, distribution, and reproduction in any medium, provided the original work is properly cited.

\begin{abstract}
Nanoporous silicon carbide is part of the important organosilicate class of low dielectric constant alloys. We report first-principles microscopic calculations of the properties of crystalline nanoporous $\mathrm{SiCH}$ systems. Properties examined include the density, pore size, dielectric constant, and strain moduli. We examined the relationship between the various properties and the amount of hydrogen in the material. In addition, the bonding topology is examined. The present results are compared with a variety of experiments.
\end{abstract}

\section{Introduction}

Nanoporous materials are interesting for a variety of applications because of their novel thermal, mechanical, electronic, and optical properties. For instance, nanoporous SiC-based alloys are employed in nanoelectronics [1-3], as well as in nanoelectromechanics [4], gas separation [5, 6], and biotechnology [7]. In highly integrated electronics, due in particular to their low dielectric constant $(\mathrm{K})$, nanoporous $\mathrm{SiC}$ materials are exploited to enhance circuit scaling [2]. New low-K dielectric materials are needed to continue the scaling of integrated circuits. In silicon-based integrated circuits, the delay time in the electrical response of the overall circuit depends on the circuit's RC time constant, which is controlled by the dielectric between each transistor around the various levels of metal interconnects, called the back-end-of-the-line (BEOL) dielectric. The BEOL dielectric needs to have several properties. A small dielectric constant is needed to limit the $\mathrm{RC}$ time constant. A candidate BEOL dielectric must also be stable to electric stress with a high breakdown voltage and a low leakage current. In addition, a BEOL dielectric must be compatible with modern silicon-based integrated circuits, that is, candidate dielectrics must be thermally and mechanically stable during silicon-integrated-circuit processing [1]. Characterizing the properties of nanoporous alloys is of fundamental and applied interest. Despite the importance of nanoporous SiC-based alloys, there is limited microscopic understanding of their physical properties.

The most common low-K SiC materials are deposited by spin-on glass technology or plasma enhanced chemical vapor deposition. As porosity increases, the alloy's dielectric constant decreases but the mechanical and electrical properties degrade $[8,9]$. The properties of the material are necessarily due to intrinsic and process-dependent factors. Experimentally, King et al. have combined several characterization techniques to investigate the electrical and physical properties of nanoporous SiC [10-13]. Theoretically, less work has been done. Recently, Calvino et al. have explored the role of pore surface passivants on the electronic properties of porous $\mathrm{SiC}$ systems $[14,15]$. Models of varying pore sizes have yet to be explored theoretically.

In this paper, microscopic models of nanoporous $\mathrm{SiC}$ are constructed to aid in the understanding of the properties of these materials. Below, several models of nanoporous $\mathrm{SiCH}$ alloys are presented for hydrogen concentrations from zero to the maximum observable value of 35 atomic $\% \mathrm{H}$. Properties compared to experiment are density, pore radius, dielectric constant, Young's modulus, and hydrogen covalent bonding ratios. The models include a single pore within a crystalline 


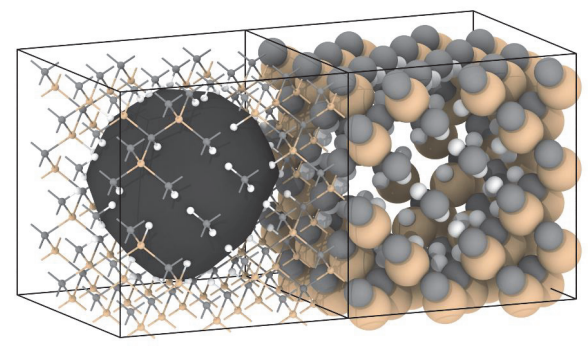

FIgUre 1: Two representations of a nanoporous SiC:H model. Balls of white, grey, and dark (brown) are for hydrogen, carbon, and silicon atoms, respectively. On the left is a ball-and-stick model with the pore region shaded black. On the right is the same model using space filling balls to represent the atoms.

$\mathrm{SiC}$ matrix and may not be expected to be a good representation of real films which involve a distribution of pores sizes within an amorphous $\mathrm{SiC}$ matrix. Nevertheless, the present models do reproduce many properties of amorphous nanoporous $\mathrm{SiC}$ suggesting these models capture important features of these materials.

\section{Models and Methods}

For these calculations, pore models are constructed from supercell structures of bulk crystalline silicon carbide polytypes, $3 \mathrm{C}-\mathrm{SiC}$ and $2 \mathrm{H}-\mathrm{SiC}$. The underlying crystalline supercells have lattice constants ranging $\sim 1.0-1.5 \mathrm{~nm}$ with between 128 and 360 atoms. Models for nanoporous $\mathrm{SiC}$ are constructed and include pores with various sizes. Starting with a $\mathrm{SiC}$ bulk periodic supercell, we remove a sphere of atoms and then passivate the dangling bonds with hydrogen in order to create single-pore $\mathrm{SiCH}$ alloy model. The bonding percentages can be adjusted by varying the center and radius of the sphere of atoms to be removed. For instance, a small sphere centered on a $\mathrm{Si}$ would result in one $\mathrm{Si}$ atom being removed. The final model would be a hydrogenated $\mathrm{Si}$ vacancy with four $\mathrm{C}-\mathrm{H}$ bonds. Of course, if the small sphere is centered on a $\mathrm{C}$ atom, then the model produced would be a hydrogenated $\mathrm{C}$ vacancy. In both cases, four hydrogens are added. Below we report the range of model properties for a given hydrogen concentration. As the sphere of the atoms removed increases, the number of hydrogens added also increases. Once the atomic structure is determined, the coordinates and the supercell volume are allowed to fully relax. Figure 1 shows an example of one specific nanoporous $\mathrm{SiC}$ model. Below we report model properties as a function of the hydrogen concentration.

For our ab initio total energy calculations, we use density functional theory (DFT) within the local density approximation (LDA) as implemented in the VASP code $[16,17]$. Core electrons are treated with the Projector Augmented Wave method [18, 19]. A plane wave energy cutoff of $300 \mathrm{eV}$ is used for the basis functions. Since we are considering large supercells with lattice constants greater than $1 \mathrm{~nm}$, only the $\Gamma$ point is used for integrations over the Brillouin Zone. Atoms and volumes are relaxed until total energy changes are less

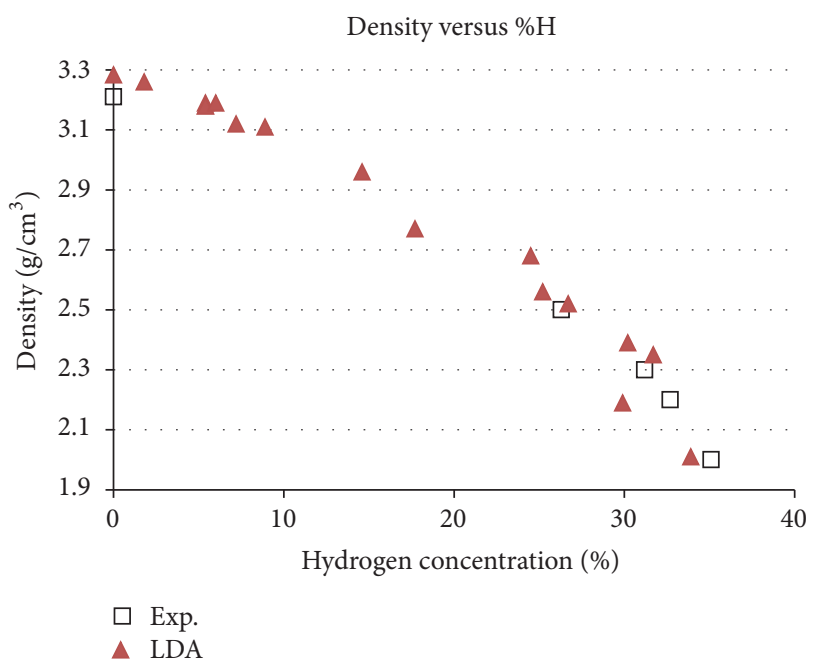

FIGURE 2: The density versus hydrogen concentration is reported. The filled (red) triangles are for the present LDA results. Open squares are for previous experimental results for nanoporous $\mathrm{SiC}: \mathrm{H}$ with $\% \mathrm{H}$ between 25 and $30 \%$ [20]. Also included is the known density for crystalline $\mathrm{SiC}$ at zero $\% \mathrm{H}$.

than $0.001 \mathrm{eV}$. Tests indicate that the structural properties reported here are well converged.

\section{Results}

The models considered here are based on crystalline SiC. However, actual nanoporous $\mathrm{SiC}$ films involve an amorphous matrix with mixed homopolar and heteropolar bonding. There are two reasons the crystalline models studied here may be good representations of amorphous nanoporous films. First, the concentration of homopolar bonding is known to be low for the range of alloys considered here [11]. Second, for the porosity of the films considered here, the $\mathrm{SiC}$ matrix is only a few layers thick in between pores, as illustrated in Figure 1. While the initial $\mathrm{SiC}$ matrix is crystalline, after the pores are created and passivated with hydrogen, the final $\mathrm{SiC}$ network does not resemble a bulk SiC crystalline material. Perhaps the $\mathrm{SiC}$ matrix is close to the actual amorphous matrix. Considering a full range of truly amorphous matrixes is beyond the scope of the present study.

The density and hydrogen concentration of the constructed pore models is the primary tool used to sort out the good quality models from the unrealistic ones. For the experimentally relevant range, between 25 and 35 atomic $\% \mathrm{H}$, the models considered for closer examination were models with densities within $0.1 \mathrm{~g} / \mathrm{cm}^{3}$ of the respective experimental value. Figure 2 shows the density of our single-pore models as a function of the atomic percentage of hydrogen in the models. The results at zero incorporated hydrogen are for crystalline SiC. Our LDA results (solid triangles) are compared to experimentally determined densities (open squares) for crystalline $\mathrm{SiC}$ and nanoporous $\mathrm{SiCH}$ [11]. The size of the data points represents the uncertainty in the density values. For crystalline $\mathrm{SiC}$, our LDA density is slightly above the experiment value because LDA underestimates the $\mathrm{SiC}$ 


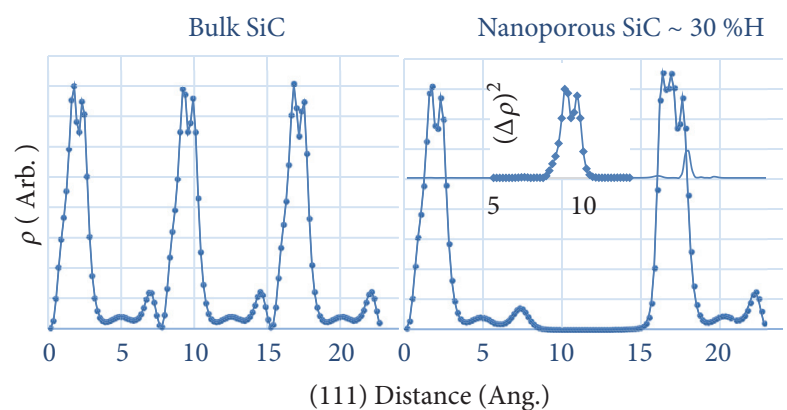

(a)

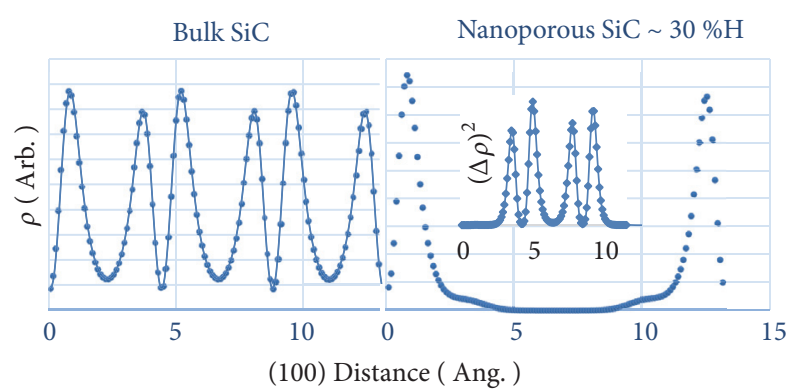

(b)

Figure 3: For the model in Figure 1, we show the charge density along two line through the center of the model and also through the corresponding crystalline $\mathrm{SiC}$. On top, the line goes diagonally along the 111 direction whereas on the bottom the line considered goes horizontally along the 100 direction. The insert on the right figure represents the charge density difference between the crystalline and nanoporous $\mathrm{SiC}$ models.

lattice constant by $\sim 1 \%$. As hydrogen is added, the density reduces mainly because atoms are being removed. However, the added hydrogen atoms repel each other leading to volume expansion. For instance, one model with 30\% incorporated hydrogen experiences a $3.5 \%$ volume expansion resulting in a $0.08 \mathrm{~g} / \mathrm{cm}^{3}$ lower density. We find a strong, almost linear, negative correlation between hydrogen concentration and density.

The hydrogen concentration is also correlated to the size of the pores in the system. Figure 2 shows porous $\mathrm{SiC}: \mathrm{H}$ densities ranging experimentally from $\sim 25$ to 35 atomic \% of hydrogen. The single-pore models have been constructed to cover the full density range from 0 to $35 \%$. Experimentally grown nanoporous $\mathrm{SiC}: \mathrm{H}$ films have a maximum hydrogen incorporation of 35 atomic \%. Theoretically, we consider hydrogenated pores within supercells with lateral dimensions $\sim 1.3 \mathrm{~nm}$ in length. At $30 \%$ hydrogen, we find that the spherical pores begin to exceed the size of the supercell creating a model of a vacuum channel instead of an isolated pore model. To consider single-pore models with hydrogen concentrations greater than $30 \%$, we use supercells with dimensions $\sim 1.5 \mathrm{~nm}$.

The pore diameters can be easily estimated from our models. The hydrogens passivating the pore surface can serve as the pore boundary. For pore models with the experimentally relevant hydrogen concentrations, between 25 and 35 atomic \%, we find the pore radii range from 4.0 to $7.0 \AA$. Reference [11] did not report pore sizes for their samples. However, in a related study by some of the same authors, pore sizes were estimated using positron annihilation lifetime spectroscopy [10]. Similar to our findings for simple singlepore models, the pore size is inversely proportional to the density of the material: lower-density materials have larger pore radii. For a nanoporous $\mathrm{SiC}: \mathrm{H}$ film with a density of $2.3 \mathrm{~g} / \mathrm{cm}^{3}$, King et al. [10] estimate a pore radius of $\sim 1.5 \AA$, much smaller than the value found from considering the hydrogen distances in the present single-pore models. The disagreement may be due to the fact that positron annihilation is caused by the interactions of positrons with electrons. The experimental pore diameter is determined by the electron cloud's depletion region.

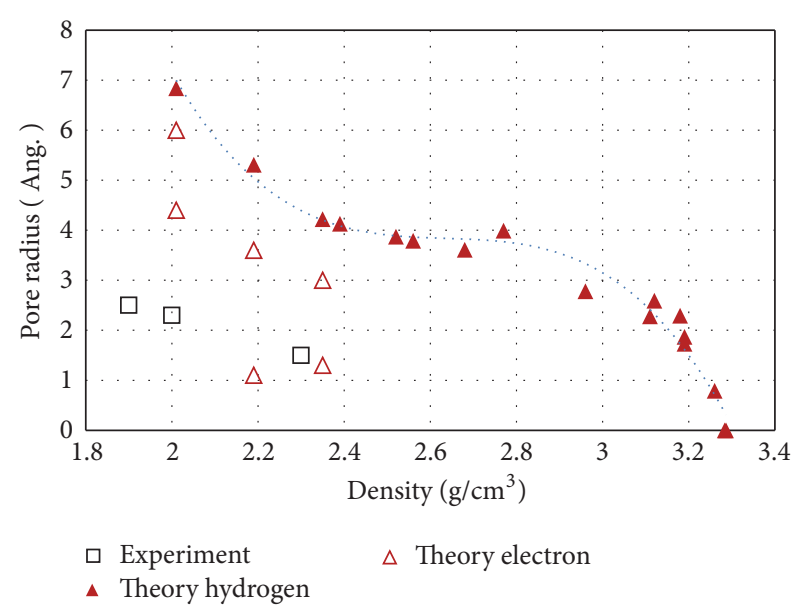

FIGURE 4: Pore radii are reported versus density. The previous experimental results are the open squares [10]. The solid (red) triangles are the present results using the hydrogen atoms to estimate the size of the pore whereas the open triangles are estimates based on the electron densities such as those shown in Figure 3.

Positron lifetimes can be calculated starting from LDA wave functions [21], but such a calculation is beyond the scope of the present study. Nevertheless, the electron density plots provide an estimate for the diameter of the evacuated region that a positron would find itself in. Figure 3(a) reports the electron density along the diagonal for a crystalline $\mathrm{SiC}$ supercell (left) and the respective nanoporous $\mathrm{SiC}: \mathrm{H}$ model (right). There are significant low charge density regions within bulk SiC. To get a better appreciation of the vacuum region of the nanoporous model, the inset shows the region where the charge density difference squared is appreciable. The radius of this region is $\sim 1.1 \AA$. However, the electron distribution varies depending on direction. In Figure 3(b), the electron density along the (100) direction is plotted for the same crystalline $\mathrm{SiC}$ supercell (left) and the same nanoporous model SiC (right). Again, the inset on the right is the charge density difference squared. The radius of the region of charge density deviations is $\sim 3.6 \AA$.

Figure 4 shows the pore radii as function of density. Experimental results are the open squares. Solid triangles 


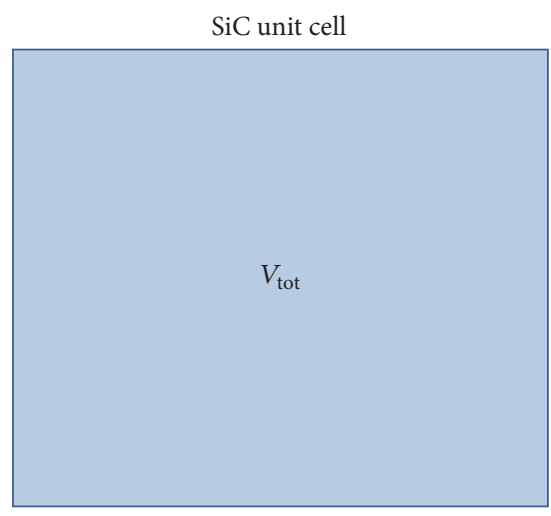

(a)

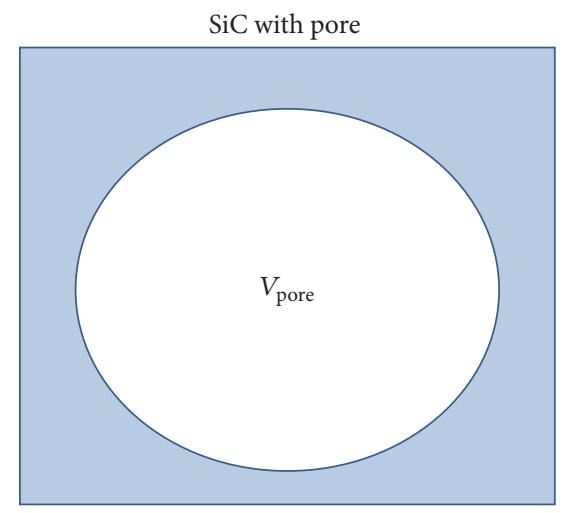

(b)

Figure 5: The unit cell is schematically shown for a crystalline (a) and nanoporous (b) SiC models. The volumes shown are used in the dielectric constant calculation.

represent the pore radii as found from hydrogen distances. The dotted line is just to guide the eye. The radii are calculated by determining the average distance between the hydrogens and the center of the pore, defined by the center of the hydrogen atoms. The open triangles are the pore radii based on electron density differences as discussed above. It is noteworthy that the range of radii estimates based on electron distributions are closer to the values reported from positron annihilation experiments. Positron annihilation lifetime calculations in c-Si finds that monovacancies and much larger tetravacancies have very similar lifetimes, suggesting that positron annihilation analysis also can underestimate the size of a pore [22], perhaps explaining the difference reported in Figure 4.

Another important aspect of nanoporous $\mathrm{SiC}: \mathrm{H}$ is the variation of the dielectric constant with hydrogen concentration or density. Figure 5 illustrates a bulk $\mathrm{SiC}$ unit cell versus a unit cell with a single pore. Applying classical dielectric theory to the unit cell with a nanopore (Figure 5(b)), the total dielectric constant $\left(K_{\mathrm{npSiC}}\right)$ can be written as $[10,23]$

$$
K_{\mathrm{npSiC}}=K_{\mathrm{SiC}} \frac{V_{\mathrm{SiC}}}{V_{\text {tot }}}+1 \frac{V_{\text {pore }}}{V_{\text {tot }}}
$$

where the dielectric constant of the solid matrix $\left(K_{\mathrm{SiC}}\right)$ may differ from the bulk SiC dielectric constant because, in the presence of a large percentage of pores, the $\mathrm{SiC}$ matrix is never more than a few atomic layers between pores. To apply the classical equation to our structural models, we need to determine a value for the pore volume. As illustrated in Figure 3, the pore radii based on hydrogen coordinates alone can overestimate the actual size of the vacuum region. In the present calculations of the dielectric constant, the model's pore diameters are chosen to be the hydrogenbased values reduced by $1 \AA$. To get a good fit between the nanoporous $\mathrm{SiC}$ experimental data [10] and the present theory, a single empirical parameter, $K_{\mathrm{SiC}}=8.3$, is used which is slightly lower than the dielectric constant of bulk $\mathrm{SiC}$. This is reasonable since the $\mathrm{SiC}$ matrix in nanoporous $\mathrm{SiC}$ involves subnanometer-thin layers of $\mathrm{SiC}$ surrounded

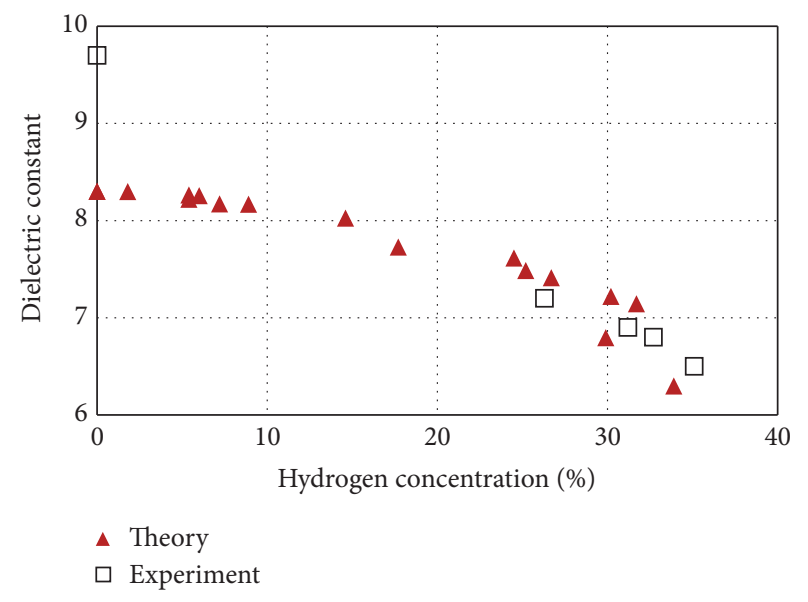

FIGURE 6: The dielectric constant is reported as a function of $\% \mathrm{H}$ in the $\mathrm{SiC}$ models. The solid (red) triangles are the present theoretical results based on classical dielectric theory and the present models. The open triangles are previous experimental values [10].

mainly by vacuum. The theoretical dielectric constant results are compared with experimental values in Figure 6.

Now let us consider the mechanical properties of our models. The bulk modulus has been calculated by examining the energy as a function of volumetric strain around the minimum and fitting the results to an equation of state. Figure 7 shows the theoretical bulk modulus for the singlepore models (solid triangles) versus atomic percent of hydrogen. The bulk modulus appears to be well approximated by a linear relation. The present theoretical $B$ value of $228 \mathrm{GPa}$ for $3 \mathrm{C}-\mathrm{SiC}$ is close to the experimental value of $224 \mathrm{GPa}$ [24]. The theoretical slope is approximately: $4 \mathrm{GPa} /$ atomic $\% \mathrm{H}$. Young's modulus can be related to the bulk modulus via the Poisson ratio $(v)$. Analysis of recent ultrasonic experiments employed a Poisson ratio of 0.25 to report experimental Young's moduli $(Y)[10]$. The relation between the two moduli is $Y=3 B(1-$ $2 v)$ which, with $v=0.25$, simplifies to $Y=1.5 B$. The present Young modulus verse density results are shown in Figure 8 as solid triangles with the experimental $Y$ values 


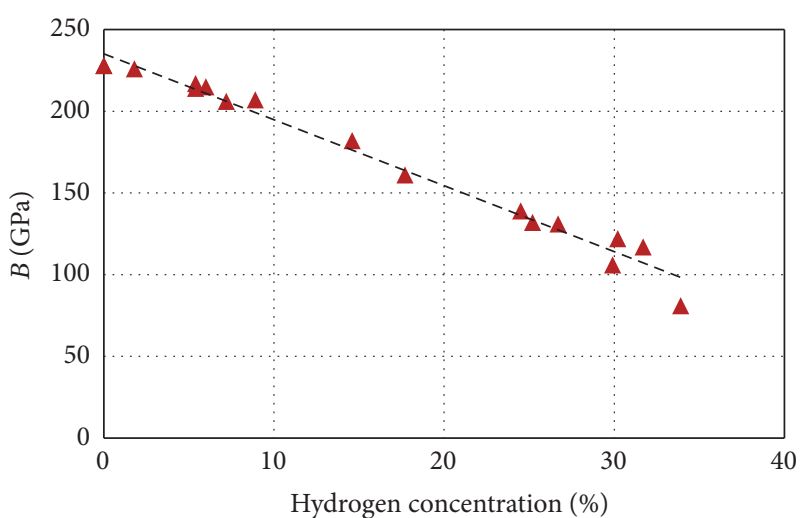

FIGURE 7: Bulk modulus and hydrogen concentration are reported for the present models from our LDA calculations. The best fit line is also shown.

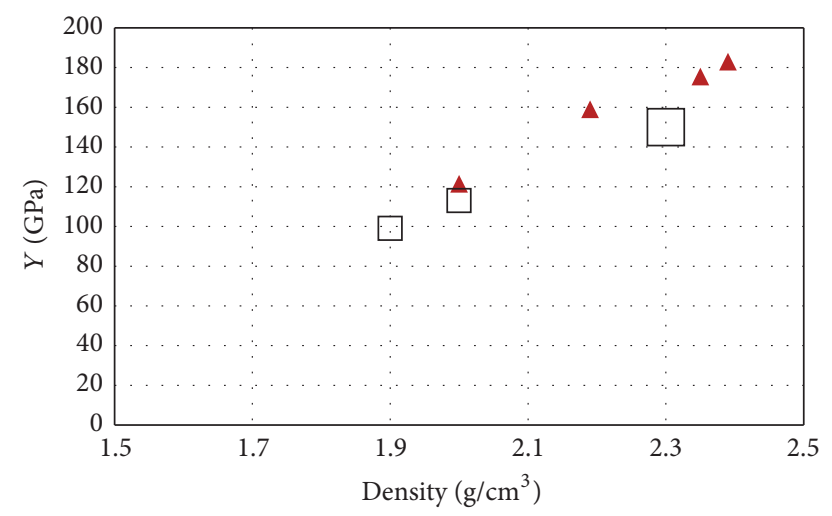

$\Delta$ LDA theory

$\square$ Experiment

FIGURE 8: Young's modulus results versus density are reported. The present LDA theoretical results are the solid (red) triangles and the open squares are the previous experimental results, where the size of the square indicates the experimental uncertainty [10].

being open squares. The size of the open squares represents the uncertainty [10]. The theoretical values reasonably match the experimental results over the experimental range.

The bonding topology is an important characteristic of nanoporous $\mathrm{SiCH}$. Since the present models are derived from bulk $\mathrm{SiC}$, there are no $\mathrm{C}-\mathrm{C}$ or $\mathrm{Si}-\mathrm{Si}$ bonds by construction. However, the $\mathrm{Si}-\mathrm{H}$ and $\mathrm{C}-\mathrm{H}$ varied depending on the pore size and location. To explore bonding topology, consider the three models with hydrogen concentration $\sim 30 \%$ and a density is $\sim 2.3 \mathrm{~g} / \mathrm{cm}^{3}$. For the three " $30 \% \mathrm{H}$ " models, C-H bonds were more prevalent than $\mathrm{Si}-\mathrm{H}$ bonds with $39 \mathrm{Si}-\mathrm{H}$ bonds to $111 \mathrm{C}-\mathrm{H}$ bonds resulting in an overall ratio of 0.35 . The majority of network atoms are bonded to one hydrogen atom: $79 \mathrm{C}-\mathrm{H}$ versus $21 \mathrm{C}-\mathrm{H}_{2}$ bonds and $35 \mathrm{Si}-\mathrm{H}$ versus 4 $\mathrm{Si}-\mathrm{H}_{2}$ bonds. The predominance of monohydride bonding found in these models is consistent with the conclusions from FTIR experiments on samples with similar densities [11]. Interestingly, the ratio ( $\mathrm{Si}-\mathrm{H} / \mathrm{C}-\mathrm{H})$ varied from 0.23 to 0.57 between the three models considered. The model with a 0.57
(Si-H/C-H) ratio has a hydrogen concentration of 29.9 atomic $\%$ and a somewhat low density of $\sim 2.2 \mathrm{~g} / \mathrm{cm}^{3}$ as reported in Figure 2. Experimental samples with densities greater than $2 \mathrm{~g} / \mathrm{cm}^{3}$ are predicted to include hydrogen with mainly C-H bonding.

\section{Summary}

Several single-pore models of nanoporous hydrogenated $\mathrm{SiC}$ have been generated. Remarkably, these simple model structures reproduce a large range of properties from recently grown samples of nanoporous SiC. Models with densities and hydrogen concentrations are found to match experiments as illustrated in Figure 2. The model pore radii versus density in the present model structures are compared with experiments in Figure 4. The theoretical pore radii due to electron distributions are close to the results of positron annihilation experiments. A simple theoretical model for the dielectric constant $(K)$ is developed using the present structural model information. In Figure 6, the model $K$ results are shown to reproduce experimental values of the nanoporous materials. The $K$ model results suggest the underlying $\mathrm{SiC}$ matrix has a dielectric value lower than bulk $\mathrm{SiC}$ as expected for nanoscale materials. In Figure 7, the bulk modulus $(B)$ is shown to vary linearly with hydrogen concentration. Young's modulus for selective models closely matches experimental values as shown in Figure 8. Finally, the $\mathrm{C}-\mathrm{H}$ and $\mathrm{Si}-\mathrm{H}$ bonding statistics are reported and found to agree with the conclusions of a recent FTIR study. Nanoporous $\mathrm{SiCH}$ prefers $\mathrm{C}-\mathrm{H}$ bonds over $\mathrm{Si}-\mathrm{H}$ bonds.

The present pore model construction method differs greatly from the experimental situation. Nevertheless, the wide range of properties reproduced by the models encourages confidence in the quality of the models. The agreement between these simple models and experiment suggests that many physical properties of these materials are due to fundamental aspects of the pore to $\mathrm{SiC}$ matrix ratio and perhaps less to process-dependent features of the amorphous materials. The present single-pore models can be the foundation for constructing complex models. In addition, the presently examined models can be the basis for new theoretical research to explore novel microscopic properties, including the role of point defects on nanoporous $\mathrm{SiC}$ dielectrics used in electronic devices.

\section{Competing Interests}

The authors declare that there is no conflict of interests regarding the publication of this paper.

\section{Acknowledgments}

This research was funded by NSF Grant RUI-DMR 1506403 (Blair R. Tuttle, Nathan J. Held, Lai Hin Lam) and by NSF Grant ECCS-1508898 at Vanderbilt University. In addition, Lai Hin Lam thanks the Vanderbilt Physics REU 2016 program for integrating him in the REU program during his stay at Vanderbilt. This research was conducted using Advanced CyberInfrastructure computational resources provided by 
The Institute for CyberScience at The Pennsylvania State University (https://ics.psu.edu/). The authors would like to thank Dr. Steve Valone for his support during this project.

\section{References}

[1] W. Volksen, R. D. Miller, and G. Dubois, "Low dielectric constant materials," Chemical Reviews, vol. 110, no. 1, pp. 56-110, 2010.

[2] A. Grill, S. M. Gates, T. E. Ryan, S. V. Nguyen, and D. Priyadarshini, "Progress in the development and understanding of advanced low $\mathrm{k}$ and ultralow $\mathrm{k}$ dielectrics for very largescale integrated interconnects-state of the art," Applied Physics Reviews, vol. 1, no. 1, Article ID 011306, 2014.

[3] S. W. King, H. Simka, D. Herr, H. Akinaga, and M. Garner, "Research updates: the three M's (materials, metrology, and modeling) together pave the path to future nanoelectronic technologies," APL Materials, vol. 1, no. 4, Article ID 040701, 2013.

[4] C. Guthy, R. M. Das, B. Drobot, and S. Evoy, "Resonant characteristics of ultranarrow SiCN nanomechanical resonators," Journal of Applied Physics, vol. 108, no. 1, Article ID 014306, 2010.

[5] B. Elyassi, M. Sahimi, and T. T. Tsotsis, "Silicon carbide membranes for gas separation applications," Journal of Membrane Science, vol. 288, no. 1-2, pp. 290-297, 2007.

[6] N. Rajabbeigi, B. Elyassi, T. T. Tsotsis, and M. Sahimi, "Molecular pore-network model for nanoporous materials. I: application to adsorption in silicon-carbide membranes," Journal of Membrane Science, vol. 335, no. 1, pp. 5-12, 2009.

[7] J.-M. Hsu, P. Tathireddy, L. Rieth, A. R. Normann, and F. Solzbacher, "Characterization of a-SiCx: $\mathrm{H}$ thin films as an encapsulation material for integrated silicon based neural interface devices," Thin Solid Films, vol. 516, no. 1, pp. 34-41, 2007.

[8] E. Van Besien, M. Pantouvaki, L. Zhao et al., "Influence of porosity on electrical properties of low-k dielectrics," Microelectronic Engineering, vol. 92, pp. 59-61, 2012.

[9] T. A. Pomorski, B. C. Bittel, C. J. Cochrane, P. M. Lenahan, J. Bielefeld, and S. W. King, "Defects and electronic transport in hydrogenated amorphous $\mathrm{SiC}$ films of interest for low dielectric constant back end of the line dielectric systems," Journal of Applied Physics, vol. 114, no. 7, Article ID 074501, 2013.

[10] S. W. King, D. Jacob, D. Vanleuven et al., "Film property requirements for hermetic low-k a-SiOxC yNz:H dielectric barriers," ECS Journal of Solid State Science and Technology, vol. 1, no. 6, pp. N115-N122, 2012.

[11] S. W. King, M. French, J. Bielefeld, and W. A. Lanford, "Fourier transform infrared spectroscopy investigation of chemical bonding in low-k a-SiC:H thin films," Journal of Non-Crystalline Solids, vol. 357, no. 15, pp. 2970-2983, 2011.

[12] S. King and J. Bielefeld, "Rigidity percolation in plasma enhanced chemical vapor deposited a-SiC:H thin films," ECS Transactions, vol. 33, p. 185, 2010.

[13] B. C. Bittel, P. M. Lenahan, and S. W. King, "Ultraviolet radiation effects on paramagnetic defects in low- $\kappa$ dielectrics for ultralarge scale integrated circuit interconnects," Applied Physics Letters, vol. 97, no. 6, Article ID 063506, 2010.

[14] M. Calvino, A. Trejo, M. C. Crisóstomo, M. I. Iturrios, E. Carvajal, and M. Cruz-Irisson, "Modeling the effects of Si-X (X=F, Cl) bonds on the chemical and electronic properties of Si-surface terminated porous 3C-SiC," Theoretical Chemistry Accounts, vol. 135, no. 4, article 104, 2016.
[15] M. Calvino, A. Trejo, M. I. Iturrios, M. C. Crisóstomo, E. Carvajal, and M. Cruz-Irisson, "DFT study of the electronic structure of cubic-sic nanopores with a C-terminated surface," Journal of Nanomaterials, vol. 2014, Article ID 471351, 7 pages, 2014.

[16] G. Kresse and J. Hafner, "Ab initio molecular dynamics for liquid metals," Physical Review B, vol. 47, no. 1, pp. 558-561, 1993.

[17] G. Kresse and J. Furthmüller, "Efficient iterative schemes for ab initio total-energy calculations using a plane-wave basis set," Physical Review B, vol. 54, no. 16, pp. 11169-11186, 1996.

[18] G. Kresse and D. Joubert, "From ultrasoft pseudopotentials to the projector augmented-wave method," Physical Review B, vol. 59, no. 3, pp. 1758-1775, 1999.

[19] P. E. Blöchl, "Projector augmented-wave method," Physical Review B, vol. 50, no. 24, pp. 17953-17979, 1994.

[20] S. W. King, L. Ross, H. Li et al., "Influence of hydrogen content and network connectivity on the coefficient of thermal expansion and thermal stability for a-SiC:H thin films," Journal of Non-Crystalline Solids, vol. 389, pp. 78-85, 2014.

[21] M. J. Puska and R. M. Nieminen, "Theory of positrons in solids and on solid surfaces," Reviews of Modern Physics, vol. 66, no. 3, article 841, 1994.

[22] D. V. Makhov and L. J. Lewis, “Two-component density functional theory calculations of positron lifetimes for small vacancy clusters in silicon," Physical Review B, vol. 71, no. 20, Article ID 205215, 2005.

[23] Z. Wu, Z. Shiung, C. Chiang et al., "Physical and electrical characteristics of methylsilane- and trimethylsilane-doped low dielectric constant chemical vapor deposited oxides," Journal of The Electrochemical Society, vol. 148, no. 6, pp. F127-F132, 2001.

[24] L. Börnstein, "Numerical data and functional relationships in science and technology, group iii: crystal and solid state physics, Vol. 13: metals: phonon states, electron states and fermi surfaces, subvolume a, phonon states of elements, electron states and fermi surfaces of alloys," Crystal Research and Technology, vol. 17 , no. 3, p. 326, 1982. 

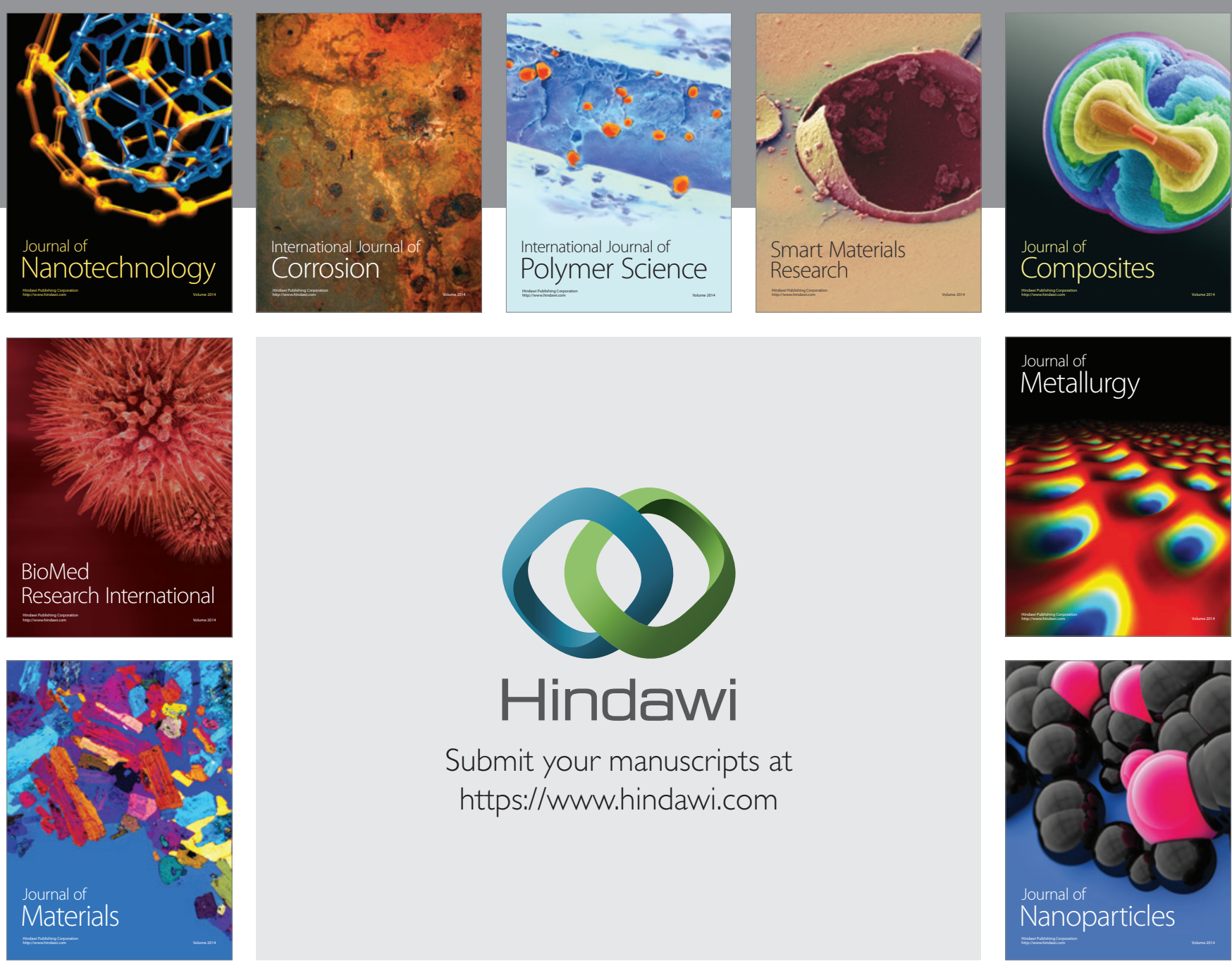

\section{Hindawi}

Submit your manuscripts at

https://www.hindawi.com

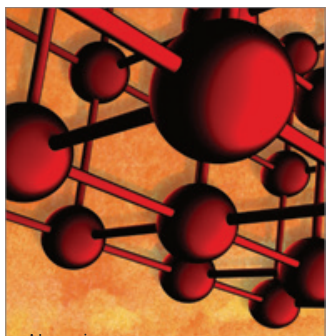

Materials Science and Engineering
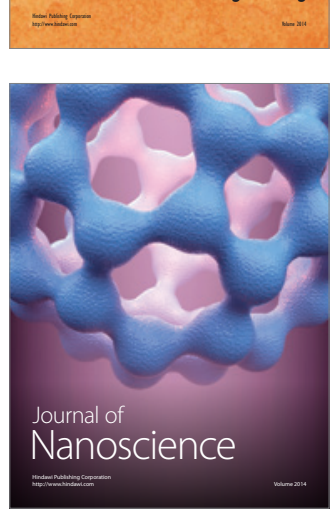
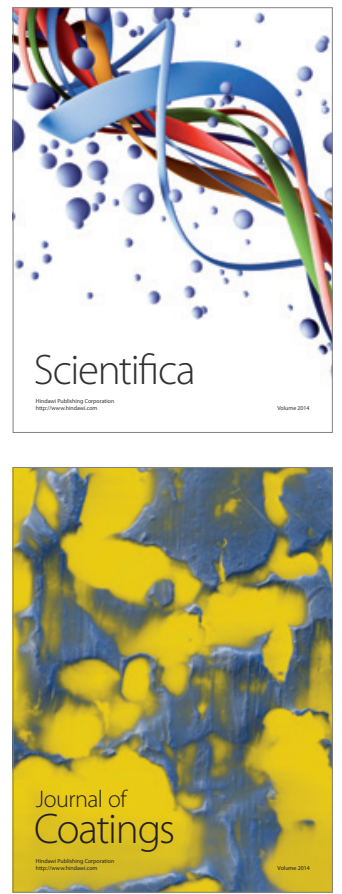
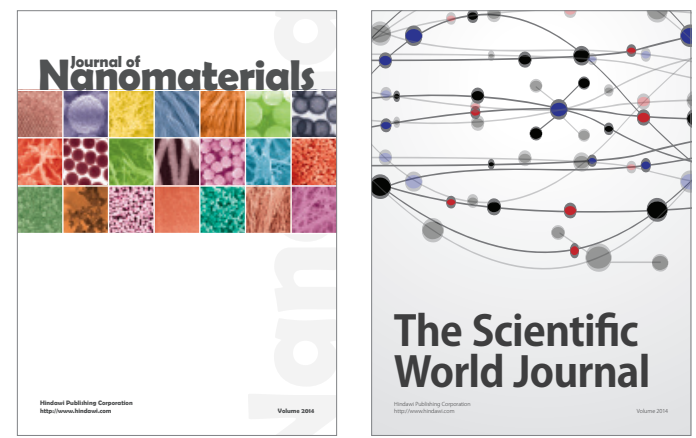

The Scientific World Journal
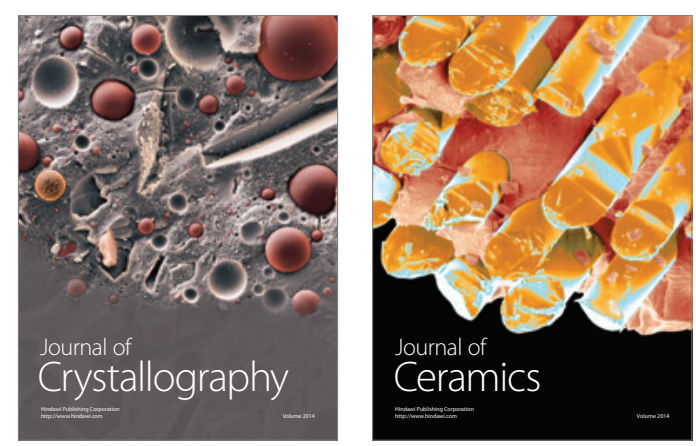
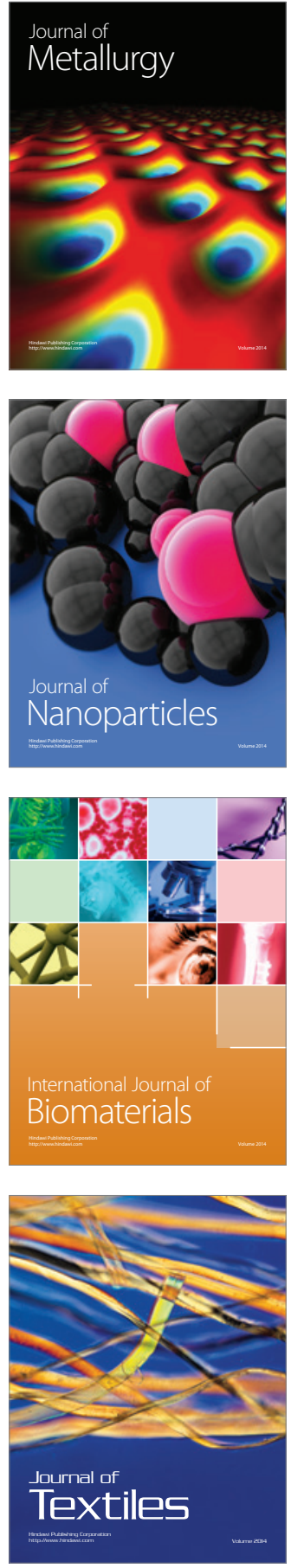